
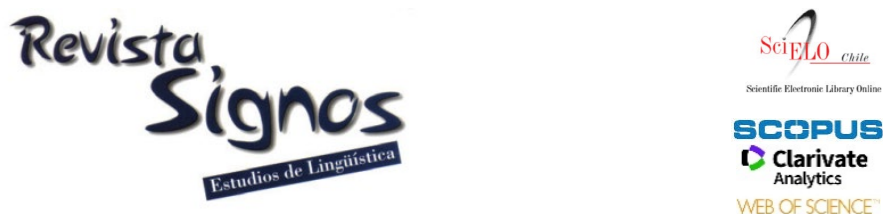

WEB OF SCIENCE

\title{
Prácticas de retroalimentación en aulas universitarias de humanidades: Comentarios digitales docentes y perfiles estudiantiles de escritor*
}

\author{
Feedback practices in Humanities university classrooms: \\ Teachers' written digital comments and students' writers profiles
}

Constanza Padilla

INVELEC/CONICET

UnIVERSIDAD NACIONAL DE TUCUMÁN

ARGENTINA

padillaconstanza@gmail.com

\author{
Esther López \\ INVELEC/CONICET \\ UNIVERSIDAD NACIONAL DE TUCUMÁN \\ ARGENTINA \\ estherlopez115@yahoo.com.ar
}

Recibido: 17-V-2018 / Aceptado: 28-VIII-2018

DOI: $10.4067 /$ S0718-09342019000200330

\section{Resumen}

Los estudios universitarios implican el ingreso a una cultura disciplinar en la que la producción de conocimientos y los modos de comunicarlos suponen un aprendizaje que debe ser enseñado en el nivel superior. En relación con esto, presentamos los resultados obtenidos con respecto a las prácticas retroalimentación entre docentes y estudiantes principiantes de Humanidades, a partir de los 'comentarios escritos digitales' (CEDs) provistos por los profesores en las sucesivas reescrituras de ponencias elaboradas por los estudiantes en un proceso gradual, con el objetivo de determinar qué clases de CEDs son más recurrentes en el corpus analizado y, en función de esto, establecer interacciones con distintos 'perfiles de escritor' (novato y experto). Los datos fueron recogidos en una asignatura de primer año de una universidad pública argentina, que participa de ciclos de investigación-acción crítica participativa, en la cual los estudiantes se inician en los modos de producción del saber, a través de la escritura de una ponencia y de su comunicación/discusión en unas Jornadas académicas. Con respecto al corpus, se han seleccionado y clasificado los CEDs provistos por un docente, en los diferentes borradores de doce ponencias estudiantiles grupales, atendiendo a categorías y subcategorías referidas al 'modo' y al 'foco'. Resultados parciales indican que habría diferencias cuantitativas en los CEDs del profesor, en relación con los diferentes perfiles de escritor, y ciertas similitudes, en las orientaciones que proporciona en cuanto a aspectos centrales del discurso académico.

Palabras Clave: Estudiantes universitarios, comentarios escritos digitales, reescritura, prácticas de retroalimentación, perfiles de escritor. 


\begin{abstract}
University studies imply the access to a disciplinary culture where knowledge production and the ways of communication it involve a learning process that needs to be taught in higher education. In connection with this, we present results obtained with regard to the feedback practices between teachers and freshmen students of Humanities, taking into account the 'written digital comments' (CEDs) provided by the teachers in the successive rewritings of presentations elaborated by the students in a gradual process. We aim to determine what kinds of CEDs are more frequent in the analyzed corpus and, depending on this, to establish interactions with different 'writer's profiles' (novices and experts), according to the criterion of the teacher-tutor. Data was gathered in a first year course from a public Argentinian university, where teachers carry out cycles of critical participative action-research, in which the students learn the ways of knowledge production, while writing a presentation and communicating/discussing it within a scientific event. Regarding the corpus, we have selected and classified the CEDs provided by a tutor in the different drafts of twelve student group presentations, attending to categories and subcategories referred to the 'mode' and to the 'focus'. Partial results indicate that there would be quantitative differences in the CEDs of the tutor, in relation to the writer's different profiles, and certain similarities, in the orientations that the tutor provides about central aspects of academic discourse.
\end{abstract}

Key Words: University students, written digital comments, rewriting, feedback practices, writer's profiles.

\title{
INTRODUCCIÓN
}

En la actualidad es ampliamente reconocida la brecha existente entre las prácticas de escritura de los estudiantes del nivel secundario y las prácticas requeridas en la universidad. En relación con esto, coexisten investigaciones que focalizan en las dificultades estudiantiles para producir textos académicos de diferente complejidad, con una clara toma de conciencia acerca de la necesidad de que la escritura académica sea objeto de reflexión y de enseñanza en este nivel (Carlino, 2013).

$\mathrm{Al}$ respecto, desde hace más de cuatro décadas, se llevan a cabo en países como Estados Unidos y Australia, diversos estudios teóricos y aplicados, a través de los conocidos movimientos WAC y WID', que se han materializado en diferentes propuestas institucionales, como por ejemplo, los 'centros de escritura' (Molina Natera, 2015), pensados como espacios fuera de las aulas para remediar dificultades escriturarias de grupos minoritarios; o bien talleres de 'escritura intensiva' al inicio de los estudios superiores, a cargo de especialistas en composición escrita, o desarrolladas al interior de las cátedras, sostenidas por los docentes de cada disciplina (Russell, 2012; Carlino, 2013). En el contexto latinoamericano las investigaciones son relativamente más recientes y han ido instalándose con mayor fuerza en las dos últimas décadas, particularmente, en países como Argentina, Chile y Colombia (Carlino, 2010; Parodi, 2010; Pérez Abril \& Rincón Bonilla, 2013; entre otros). 
Un grupo importante de investigaciones actuales señala la necesidad de implementar estas propuestas de enseñanza en contextos dialógicos, que permitan repensar las tradicionales prácticas de retroalimentación escrita (en inglés, written feedback), centradas mayormente en una relación unidireccional docente-estudiante. Estos nuevos enfoques remarcan la necesidad de favorecer intercambios multidireccionales entre estudiantes y docentes, y entre pares (Nicol, 2010; Nicol, Thomson \& Breslin, 2014), que posibiliten interactuar no solo a través de los comentarios escritos, sino también en espacios de intercambios orales presenciales o mediados por la tecnología (Alvira, 2016). Esto supone una conceptualización de la producción escrita, en tanto proceso recursivo y la focalización en un aspecto sustancial de este como lo es la revisión. En este sentido, se han intensificado las investigaciones didácticas que se centran en las prácticas de revisión colaborativa, tanto entre pares, como entre docentes y alumnos (Álvarez Angulo, 2011; Cano, Corcelles, Castelló \& Fuentealba, 2012; Aguilera \& Boatto, 2013; Corcelles, Cano, Bañales \& Vega, 2013).

En el contexto anglófono, los estudios sobre feedback vinculados con el mejoramiento de la escritura, tienen una larga tradición fundamentalmente referida a la enseñanza del inglés como lengua extranjera o segunda (Lillo Durán \& Ferreira Cabrera, 2014). Algunas investigaciones dan cuenta del papel crucial que tienen los comentarios escritos para el mejoramiento de los textos, al afirmar que contribuyen a que los estudiantes tomen gradualmente el control de su propio aprendizaje hasta llegar a la autorregulación (Nicol \& Macfarlane-Dick, 2006); en tanto otras ponen en duda el efecto positivo de estos comentarios (Truscott, 1996), ante la falta de estudios empíricos contundentes. Por su parte, en las últimas décadas han cobrado relevancia los estudios sobre la influencia de la tecnología en las prácticas de feedback (GarcíaYeste, 2013), considerando sus ventajas y desventajas con respecto a los soportes tradicionales.

Otra línea de investigación que ha comenzado a visibilizarse en los últimos años es la que tiene en cuenta la importancia de considerar la singularidad de cada estudiante, a fin de proveer una retroalimentación adaptada a sus necesidades. En tal sentido, a partir del concepto de 'perfiles de escritor' (Creme \& Lea, 2000), algunas investigaciones en curso plantean la conveniencia de proporcionar comentarios diferenciales, en función de estos (Pardo \& Castelló, 2016).

Con el propósito de aportar a la discusión acerca de alternativas didácticas para trabajar con la escritura académica en las aulas universitarias, en este artículo, nos proponemos poner en relación prácticas de retroalimentación docente-materializadas a través de comentarios escritos digitales (en adelante, los CEDs) - con perfiles estudiantiles de escritor, definidos en función de su rendimiento académico y de sus trayectorias previas. 
Los datos fueron obtenidos en una asignatura de un primer año de Humanidades de una universidad pública argentina -que participa de ciclos de investigación-acción-, en la que se implementa un proyecto de escritura académica auténtica (la elaboración gradual de una ponencia y su socialización en unas jornadas académicas), con un fuerte apoyo de prácticas de retroalimentación oral y escrita, que permite a los estudiantes no solo aprender a escribir académicamente en un proceso recursivo -con numerosos borradores-, sino también inscribirse como productores principiantes del conocimiento disciplinar.

En esta oportunidad, nos centramos en los CEDs proporcionados por un docente, a las diferentes versiones escritas de doce ponencias grupales, realizadas por estudiantes de dos cohortes de la citada asignatura. Para su análisis, seleccionamos categorías de diferentes clasificaciones (Straub \& Lunsford, 1995; Duijnhouwer, 2010; entre otros), a fin de construir una clasificación integrada, a partir dos criterios generales (modo y foco).

En tal sentido, nos hemos propuesto los siguientes objetivos: 1) Determinar qué clase de CEDs son más recurrentes en el corpus analizado. 2) Identificar si el docente formula diferentes tipos de CEDs, en función de distintos perfiles de escritor. 3) Establecer si, más allá de estas diferencias, pueden detectarse similitudes en los CEDs, en relación con los desafíos que plantea la complejidad de la escritura académica.

En relación con estos objetivos, pretendemos profundizar en la comprensión de las interacciones que se dan entre los procesos de escritura de las ponencias estudiantiles y las mediaciones didácticas que, si bien plantean problemas desafiantes a los estudiantes, regulan todas las alternativas del proceso, a través de apoyos sistemáticos que promueven la revisión de los borradores. En particular, nos interesa ahondar en el diferente interjuego que se produce entre los procesos de 'andamiaje' (Bruner, 1984) y de 'autorregulación' (Zimmerman, 2000) en los grupos, de acuerdo con los diferentes perfiles estudiantiles.

En esta oportunidad, testeamos la siguiente hipótesis en el corpus seleccionado: existe una relación entre la cantidad y el tipo de CEDs proporcionados por el docente y diferentes perfiles de escritor, en relación con el grado de dominio de la escritura a nivel general (coherencia global y local, y aspectos normativos) y a nivel de las restricciones específicas del discurso académico.

A continuación, nos detendremos en algunos antecedentes que dan cuenta del estado actual de las investigaciones sobre las prácticas de feedback y las conceptualizaciones sobre perfiles de escritor. Luego, explicitaremos los conceptos teóricos que vertebran la investigación y el marco metodológico seleccionado para la recolección, análisis e interpretación de los datos. En relación con los objetivos planteados, consideraremos los resultados obtenidos y los pondremos en diálogo con otros datos de investigación. Por último, ofreceremos una breve síntesis de las 
principales fortalezas y limitaciones del presente estudio, lo que permitirá, a la vez, plantear futuras líneas de investigación.

\section{Antecedentes}

Como señalamos en la introducción, la visibilización de los estudios sobre escritura académica responde a la necesidad de avanzar en propuestas didácticas que favorezcan la apropiación de la cultura escrita en el marco de las comunidades disciplinares. Una de las formas, a través de las cuales se ha promovido la enseñanza sostenida de la escritura, ha sido fomentar las prácticas de retroalimentación entre el docente y sus estudiantes. Si bien en el contexto anglófono existe una larga tradición de investigaciones sobre el feedback y sus aplicaciones en la enseñanza, en el contexto español y latinoamericano, tanto los estudios teóricos como aplicados en el nivel universitario, se han desarrollado en la última década (Corcelles, et al. 2013; Corcelles \& Oliva, 2016; Tapia-Ladino, Arancibia \& Correa, 2016; Corcelles, Cano, Mayoral \& Castelló, 2017), quizás debido a que se conocieron, valoraron y adoptaron tardíamente los difundidos modelos de producción escrita que privilegian su carácter procesual y recursivo (Flower \& Hayes, 1981; Scardamalia \& Bereiter, 1992).

Con respecto a las principales discusiones que se han planteado sobre las prácticas de feedback, podemos sintetizarlas en tres grandes líneas de investigación: las referidas a sus grados de efectividad, atendiendo tanto a las perspectivas de docentes y estudiantes, como a la calidad final de los escritos logrados; las que focalizan en el impacto de las TICs como mediadoras de estas prácticas, y las que se centran en las tipologías de los comentarios escritos, uno de los recursos característicos de las tareas de retroalimentación.

En relación con la primera línea de investigación, el debate se remonta a mediados de los años 90 del siglo pasado, cuando Truscott (1996), al referirse a los estudios sobre la enseñanza del inglés como lengua extranjera, pone en duda la efectividad del feedback correctivo escrito (centrado en los aspectos formales de la escritura), e incluso plantea su nocividad. Frente a esta posición, Ferris (1999) sostiene la importancia de la retroalimentación, ya que es requerida por los estudiantes quienes necesitan responder a la exigencia de precisión gramatical demandada por sus profesores. En la actualidad, esta postura favorable hacia el uso del feedback escrito es mayormente aceptada (Peterson, 2010; Nicol et al., 2014; entre otros), enfatizando que esta práctica es valorada positivamente por los estudiantes (Tapia-Ladino, Bustos, Salazar, Muñoz, Varela \& Sáez, 2014), ya que permite tanto brindar un apoyo sostenido en el aprendizaje de la escritura, como mejorar su rendimiento posterior, favoreciendo su autorregulación. En relación con esto, Peterson (2010) considera que los alumnos toman conciencia del poder de la escritura, cuando reciben retroalimentación por parte de sus pares o profesores, porque comprenden lo que el lector ha experimentado 
frente a su texto. Además, les permite constatar en qué medida su escrito responde a las expectativas genéricas de su comunidad disciplinar.

En conexión con el debate acerca de la efectividad de las prácticas de feedback, en los últimos años se centró en la discusión sobre la incidencia de las TICs en los modos de proporcionar retroalimentación. En este sentido, algunos autores como Tuzi (2004) plantean las ventajas de los comentarios electrónicos, con respecto a los tradicionales en papel, por los efectos positivos que produce en los estudiantes, ya que los insta a realizar más revisiones sobre sus borradores, gracias al medio digital que favorece de forma dinámica las tareas de reescritura.

Sin embargo, el concepto de retroalimentación electrónica no se refiere solo a los comentarios que proporciona un ser humano a través de la tecnología: por ejemplo, la opción 'control de cambios' o la opción 'insertar comentarios' del procesador Word (la primera de ellas, considerada más invasiva o menos respetuosa de la voz del escritor estudiantil, que la segunda, según García-Yeste, 2013), u otras opciones más recientes como el screencast que brinda feedback asincrónico con el apoyo de imágenes de video y voz (Alvira, 2016). También incluye programas automáticos, con distintos grados de margen para la intervención personalizada del docente. Algunos disminuyen drásticamente esta posibilidad, como los scripts de retroalimentación que incluyen un banco de comentarios electrónicos, pretendiendo aliviar las demandas laborales del docente. Algunas investigaciones señalan que los estudiantes valoran este enfoque más mecanicista porque proporciona mayor cantidad de información (Case, 2007). Sin embargo, otros autores remarcan las limitaciones de estos sistemas porque, al estar prediseñados, no permiten la interacción con un público específico, cuestión crucial tratándose de la escritura académica, en la que son fundamentales el enunciador, destinatario, contexto y propósito (García-Yeste, 2013).

Por otra parte, un volumen importante de investigaciones se ocupa de las tipologías de comentarios escritos, atendiendo a diferentes criterios: unos hacen referencia al modo en que el docente provee el feedback (directos o indirectos; positivos o negativos); otros, obedecen a los aspectos en que se focaliza la retroalimentación (cuestiones formales o de contenido). En tal sentido, estos criterios se valoran en función de características generales de las 'buenas prácticas' de feedback, como es el caso de Nicol y Macfarlane-Dick (2006), quienes postulan siete principios generales (ayuda a aclarar qué es un buen desempeño; facilita el desarrollo de la autoevaluación; brinda información de alta calidad; alienta el diálogo; estimula las motivaciones positivas y la autoestima; ofrece oportunidades para cerrar la brecha entre el desempeño actual y el deseado; proporciona a los docentes información para ser capitalizada en los modos de enseñanza).

En relación con estos principios, Nicol (2010) plantea tres líneas de investigación sobre feedback, desarrolladas en las últimas décadas: una se centra en la relación 
unidireccional docente-alumno y prioriza los comentarios directivos sobre cuestiones formales. Otra línea destaca la importancia del rol activo del estudiante, por lo cual se prefieren comentarios de tipo indirecto para favorecer el descubrimiento y el pensamiento reflexivo. La tercera línea, en la que se inscribe el propio Nicol (2010), privilegia una perspectiva dialógica, que implica prácticas de retroalimentación adaptativas, interactivas y reflexivas, tanto entre docente y alumno, como entre pares, con el objetivo de ayudar a los estudiantes a desarrollar los procesos de autorregulación de los aprendizajes.

De este modo, a la luz de estos siete principios, Nicol (2010) propone un conjunto de recomendaciones para proveer buenos comentarios (orales o escritos; presenciales o virtuales), sobre la base de investigaciones centradas en lo que valoran positivamente los estudiantes. En tal sentido, se destacan los siguientes atributos de un buen comentario: comprensible, selectivo, específico, oportuno, contextualizado, sin prejuicios, equilibrado, transferible y personal. Sin embargo, resulta difícil llegar plenamente a cubrir todos estos atributos de los comentarios en las prácticas de feedback del nivel superior, dada la masividad por la que atraviesan las universidades. Al respecto, Nicol (2010) señala que un punto de partida fundamental es conocer la trayectoria previa de los estudiantes (nivel de competencia escrita, reacción emocional ante la retroalimentación, entre otros). Al respecto, recupera los aportes de una investigación de Orsmond y Merry (2009), quienes establecen una diferencia entre estudiantes de bajo y alto rendimiento: los primeros comprenden más literalmente los comentarios escritos de sus profesores; en cambio, los segundos logran una comprensión más inferencial, buscando el significado detrás del mensaje.

De esto se deriva la necesidad de prestar especial atención a las características individuales de los estudiantes al momento de proveer retroalimentación. De allí, la relevancia del concepto de 'perfiles de escritor', conceptualizado originalmente como estilos individuales de escritura (Creme \& Lea, 2000) y retomado en algunas investigaciones en curso, para dar cuenta de cómo operan los escritores con los comentarios escritos de sus docentes y pares, en los procesos de revisión colaborativa (Pardo \& Castelló, 2016). Relacionado con esto, algunos autores esbozan la idea de proveer comentarios diferentes en función, tanto del grado de dominio de la escritura estudiantil, como de su conocimiento acerca las prácticas de feedback (tipos de comentarios y modos de responder a estos). Para ello, Alvira (2016) apunta la utilidad de los comentarios directos en la etapa inicial de las prácticas de retroalimentación y la conveniencia de los comentarios indirectos, cuando los estudiantes ya están familiarizados con estas prácticas. Por su parte, Nicol (2010), frente a la queja de los docentes sobre la falta de un conocimiento compartido profesor-alumno que favorezca una mejor retroalimentación, recurre al principio de contingencia, para adaptar los comentarios escritos a las necesidades estudiantiles (específicamente, en función de sus grados de experticia). 
En congruencia con todos estos planteamientos, en este artículo nos centramos en las prácticas de retroalimentación docente, desde un contexto dialógico (Nicol, 2010; Boucher, 2015), en relación con diferentes 'perfiles de escritor'. Para ello, en esta oportunidad, focalizamos en los comentarios digitales escritos de un docente -en adelante, los CEDs-, sin desconocer que estos constituyen uno de los recursos de estas prácticas de feedback. Por su parte, para los 'perfiles de escritor' -como veremos en el siguiente apartado-, recuperamos los aportes del modelo de composición escrita de Scardamalia y Bereiter (1992), en relación con la distinción entre escritores novatos y expertos.

\section{Marco teórico}

Nuestro encuadre conceptual se sustenta en la conceptualización de la escritura en tanto proceso cognitivo-recursivo que implica tareas sostenidas de revisión y de reescritura (Flower \& Hayes, 1981; Scardamalia \& Bereiter, 1992; Flower, 1994; Hayes, 1995).

De los aportes de Scardamalia y Bereiter (1992), recuperamos particularmente dos conceptualizaciones: la 'dimensión epistémica' de la escritura y la distinción entre 'escritores novatos' y 'expertos'. El primer concepto alude a las transformaciones cognitivas que posibilita la revisión recursiva de las producciones escritas, gracias a que se plantea la escritura como un doble espacio-problema a resolver: un 'espacioproblema retórico' y un 'espacio-problema de contenido'. En relación con esto, resulta operativa la diferencia entre 'escritores novatos' y 'expertos', en cuanto a los modos de operar con el texto en proceso, en tanto son estos últimos escritores los que logran aprovechar las potencialidades epistémicas de la escritura, ya que son conscientes de la provisionalidad del proceso escriturario. En tal sentido, retomamos esta distinción para plantear el concepto de 'perfiles de escritor' (Creme \& Lea, 2000), entendiéndolo en función del mayor o menor dominio que los estudiantes principiantes van manifestando en sus primeras experiencias de escritura académica. De allí que consideremos las categorías de 'novato' y 'experto', pero teniendo en cuenta que en ningún caso se trata de escritores expertos, en sentido pleno.

En cuanto a los aportes de Hayes (1995), rescatamos la estrecha relación que establece entre lectura, escritura y revisión, que se materializa en la doble conceptualización sobre 'leer para comprender', que proporciona información para escribir, y 'leer para corregir', que se centra en el eje de la revisión y apunta tanto al contenido como a la identificación de problemas de superficie. Al mismo tiempo, esta posibilidad del escritor de 'leer para comprender' le permite interpretar las directivas recibidas por el docente y producir un texto conforme a los requerimientos discursivos.

En relación con la recursividad textual, tomamos particularmente los aportes del modelo 'comparar, diagnosticar y operar' de Scardamalia y Bereiter (1983), quienes 
prestan especial atención a la revisión, en tanto subproceso que se da en cualquier momento de la producción escrita. Sin embargo, se plantean diferencias en los modos de recurrir a esta en los escritores novatos y expertos. Al respecto, este modelo implica poder cotejar la distancia entre el texto producido y los objetivos de escritura; determinar las causas de estas diferencias y actuar en consecuencia. Los escritores expertos son los que pueden realizar estas operaciones con mayor autonomía, en tanto los novatos requieren de un apoyo específico para desplegarlas de manera productiva; es decir, para introducir cambios sustanciales que mejoren la calidad textual y no solo cambios superficiales.

Un concepto central, en el camino para lograr el dominio de la escritura por parte de los estudiantes, es el de 'aprendizaje colaborativo' (Bruffee, 1998), entendido como un proceso en donde las personas negocian y comparten significados, orientados por la resolución de problemas. De este modo, se plantea que la colaboración ayuda a los estudiantes a aprender más profundamente, de manera más eficiente de lo que aprenden solos. Dentro de las diversas posibilidades de colaboración en las aulas (escritura colaborativa y revisión colaborativa entre pares; escrituras metacognitivas para fortalecer el conocimiento docente-alumno, entre otras), asumimos el feedback como una práctica de revisión colaborativa asimétrica, a través de la cual el docente proporciona distintos tipos de 'andamiaje' (Bruner, 1984), mediante diferentes clases de CEDs, atendiendo a los 'perfiles de escritor', en una relación de reciprocidad en la que el estudiante también se compromete a considerar de manera crítica estos comentarios y a capitalizarlos para la mejora de sus escritos en un creciente proceso de autorregulación (Zimmerman, 2000; Nicol \& Macfarlane-Dick, 2006)

En relación con los CEDs, adoptamos la conceptualización que los entiende como 'géneros pedagógicos' (Bazerman, 2004) cuya finalidad es lograr la consecución de otro género (Tapia-Ladino et al., 2016), en nuestro caso, el género académico 'ponencia'. Estos comentarios son textos de variada longitud (aunque mayormente breves) que se refieren tanto a cuestiones de estructura textual y contenido, como a aspectos normativos (gramaticales y ortográficos).

Con respecto a los tipos de comentarios, si bien hay diversas clasificaciones (TapiaLadino, 2014), seguimos parcialmente las propuestas de Straub y Lunsford (1995) y de Duijnhouwer (2010), entre otros. Para ello, partimos de la distinción entre 'modo' y 'foco', para organizar los diversos comentarios escritos, teniendo en cuenta, por un lado, la relación que se establece entre el enunciador y el destinatario ('modo') y, por otro lado, el objeto de análisis al cual apunta el comentario escrito ('foco'). En el apartado siguiente, haremos referencia con más detalle a esta clasificación, como así también al contexto de la investigación, a través del cual se recogieron los datos. 


\section{Marco metodológico}

Como ya anticipamos en la introducción, en esta oportunidad, presentamos resultados en curso obtenidos, a partir de ciclos de investigación-acción crítica participativa (Kemmis, 2009), desarrollados en una asignatura ${ }^{2}$ de un primer año de Humanidades de una universidad pública argentina -desde hace más de una década-, en la que se propone a los estudiantes un proyecto de escritura académica genuina (elaboración y socialización académica de una ponencia gestionada grupalmente). A partir de un eje común -el estudio de los 'géneros discursivos' (Bajtín, 1982) de circulación actual-, los alumnos eligen uno de ellos, de acuerdo con sus intereses, para desarrollar un estudio teórico y empírico, que implica un trabajo riguroso con fuentes bibliográficas y un proceso de indagación de la realidad, en relación con las prácticas discursivas de este género en el contexto social (modos de comprensión y/o producción de los actores sociales).

Este proyecto didáctico se realiza gradualmente durante el segundo cuatrimestre del año académico, y está precedido por una primera etapa en la que se priorizan la producción y la revisión colaborativa en parejas de un artículo mediático de opinión. En cambio, para la producción de la ponencia se plantea una escritura colaborativa más compleja (realizada por un promedio de 4 estudiantes) y la revisión de los diferentes borradores queda prioritariamente bajo responsabilidad del docente, quien además despliega diferentes prácticas de retroalimentación: los CEDs que se insertan en los diferentes borradores digitales; intercambios asiduos por correo electrónico, en los cuales los estudiantes van planteando dudas o aclaraciones sobre los procesos de investigación y escritura; discusiones en pequeños grupos y en el grupo clase sobre los CEDs recibidos.

Optamos por la investigación-acción crítica participativa (Kemmis, 2009), por cuanto es una metodología cualitativa que busca una interpretación de la realidad en profundidad, no solo a partir de la indagación sobre las prácticas y representaciones de los principales actores sociales involucrados (en este caso, docentes y estudiantes), sino también a través de la planificación e implementación de acciones transformadoras, tendientes a encontrar soluciones a los problemas que se detectan y evaluar las consecuencias de estas acciones para determinar nuevos problemas de investigación (Kemmis, 2009), en un proceso autocrítico, cíclico e interactivo que compromete a los docentes como investigadores de sus propias prácticas profesionales.

Para la recolección y el análisis de los datos seguimos el enfoque interactivo y realista-crítico de investigación cualitativa (Maxwell, 2012); en particular, las estrategias de validez para otorgar confiabilidad a este enfoque metodológico ('participación intensiva a largo plazo', 'demanda de retroalimentación a los participantes de la 
investigación', ‘búsqueda de evidencias discrepantes', ‘comparación', ‘triangulación de datos' y 'cuasi-estadísticas').

Con respecto a la 'participación intensiva a largo plazo', cabe aclarar que los ciclos de investigación-acción vienen desarrollándose desde hace trece años, en los que hemos recogido un vasto conjunto de datos en diferentes cohortes estudiantiles, obtenidos a través de diversas técnicas de recolección, tales como observaciones de clases, entrevistas a estudiantes y profesores, y recopilación de abundantes materiales escritos (correos electrónicos entre docentes y estudiantes, 'autobiografías estudiantiles de escritura', planes o bosquejos de las ponencias, sus abstracts y sus diferentes borradores, entre otros). En esta oportunidad, hemos seleccionado doce ponencias grupales de las dos últimas cohortes (con un promedio de seis borradores cada una; o sea, 72 borradores aproximadamente), supervisadas por un profesor: seis ponencias de la cohorte 2015 (con un total de 531 CEDs) y seis ponencias de la cohorte 2016 (con un total de 399 CEDs). Cabe aclarar que cada ponencia en su versión final tiene un promedio de 6000 palabras, equivalente a 15 páginas aproximadamente (sin tener en cuenta los anexos).

El criterio de selección tanto del docente como de los grupos dentro cada cohorte, fue intencional. En el caso del profesor, se lo eligió porque sus modos de retroalimentación se corresponden, en gran medida, con las buenas prácticas de feedback postuladas por Nicol (2010), en particular, en cuanto a su sistematicidad, frecuencia, calidad y grado de adaptación de los comentarios, en función de la heterogeneidad de sus estudiantes. Con respecto a los grupos de estudiantes, se los seleccionó teniendo en cuenta dos grandes criterios, derivados de las entrevistas realizadas al docente y de los datos evaluativos proporcionados por él (calificaciones de dos exámenes parciales y del artículo de opinión mediática, y datos obtenidos de las autobiografías de escritura, que dan cuenta de las trayectorias previas). Sobre la base de esta información que permite postular diferentes dominios de la escritura, se acordó con el docente distinguir los perfiles de 'experto' y 'novato', sin desconocer que hay varios alumnos que se encuentran en niveles intermedios. El primer perfil manifiesta un muy buen dominio de la escritura a nivel de coherencia global y local, y de aspectos normativos, que lo habilitan a afrontar más exitosamente los desafíos de la escritura académica. El segundo presenta limitaciones en todos estos niveles, lo que vuelve mucho más complejo el proceso de apropiación del discurso académico.

En cuanto a la 'demanda de retroalimentación a los participantes de la investigación', se priorizó la comunicación con el docente seleccionado, a fin de que brindara datos complementarios, necesarios para ponderar el análisis del corpus seleccionado.

Con respecto a la 'búsqueda de evidencias discrepantes', hemos planteado inicialmente una clasificación de los CEDs provistos por el profesor, que luego fue 
refinándose a partir del análisis exhaustivo del corpus y de su validación, a través de la evaluación de dos pares expertos. Esto permitió identificar algunas inconsistencias o solapamientos en las categorizaciones iniciales.

En lo referido a la 'comparación', hemos contrastado los diferentes tipos de CEDs del docente, para ponerlos en relación con los perfiles postulados de novato y experto. En lo que respecta a la 'triangulación de datos', si bien hemos centrado nuestros análisis en los CEDs, hemos considerado también otros conjuntos de datos tales como las autobiografías de escritura y las entrevistas al docente, para establecer interrelaciones entre los tipos de CEDs, las perspectivas estudiantiles sobre sus propias fortalezas y limitaciones en la escritura y los puntos de vista del docente en cuanto a los tipos de ayuda necesarios, de acuerdo con los perfiles de escritor.

Por último, en lo que concierne a las 'cuasiestadísticas', si bien el enfoque asumido es fundamentalmente cualitativo, hemos considerado conveniente complementarlo con cuantificaciones que permitan identificar ciertas recurrencias que serán profundizadas en estudios posteriores.

Con referencia a los tipos de CEDs, como ya anticipamos, seguimos parcialmente las propuestas de diferentes autores (Straub \& Lunsford, 1995; Duijnhouwer, 2010; entre otros). En este sentido, consideramos el 'modo' (la relación que establecen los CEDs entre enunciador-estudiante y destinatario-docente) y el 'foco' (los aspectos del texto que el docente señala para su mejoramiento). En el primer caso, tenemos en cuenta si se trata de un acto de habla directo o indirecto y la modalidad que adopta (orden, interrogación, declaración afirmativa o negativa, construcción condicional, construcción impersonal y ofrecimiento de alternativa discursiva). En cuanto al objeto de análisis, atendemos a cuestiones de diagramación, coherencia global (partes canónicas, articulación entre partes y comentarios críticos sobre el tema de investigación); coherencia local (estructura gramatical, puntuación y reformulación); registro académico (pautas de citación, uso adecuado de terminología general y de terminología específica, y posicionamiento enunciativo) y ortografía.

A continuación, incluimos, a modo ilustrativo, ejemplos de algunas categorías ${ }^{3}$. Para ello, transcribimos el fragmento objeto de revisión y el CED realizado por el docente: 


\section{MODO}

\section{Acto de habla directo (Orden)}

\begin{tabular}{|l|l|}
\hline \multicolumn{1}{|c|}{ Texto fuente } & \multicolumn{1}{c|}{ Comentario escrito digital } \\
\hline $\begin{array}{l}\text { Con respecto a los antecedentes, luego de informarnos, } \\
\text { notamos que existe un amplio campo de investigación, tanto } \\
\text { sobre el género publicitario como de la importancia de la } \\
\text { lectura, pero existe un espacio vacante sobre la publicidad } \\
\begin{array}{l}\text { como factor influyente en la lectura de los adolescentes, es } \\
\text { decir, ausencia de una investigación que relacione ambos } \\
\text { temas. }\end{array}\end{array}$ & $\begin{array}{l}\text { Aquí tenían que insertar el nombre de } \\
\text { los autores entre paréntesis como cita no } \\
\text { integrada. }\end{array}$ \\
(Ponencia 1, 2015; versión 1, CED 4)
\end{tabular}

\section{Acto de habla indirecto (Condicional)}

\begin{tabular}{|l|l|}
\hline \multicolumn{1}{|c|}{ Texto fuente } & \multicolumn{1}{c|}{ Comentario escrito digital } \\
\hline Para la especialista "hay una condición esencial para que se & $\begin{array}{l}\text { Está muy alejada Ramírez Gelbes, } \\
\text { deberían nombrarla de nuevo junto con }\end{array}$ \\
produzca el fenómeno": "Se le hace un guiño a un & el año y el n de página al ser una cita \\
interlocutor, pero solo aparece en determinadas & directa. \\
circunstancias. Debe ser un par, existe una jerarquía. A nadie & (Ponencia 4, 2016; versión 4, CED 25). \\
se le ocurriría mandarle un 'ok con la manito' a un jefe". & \multicolumn{2}{|l}{} \\
\hline
\end{tabular}

\section{Acto de habla indirecto (Ofrecimiento de alternativa discursiva)}

\begin{tabular}{|l|l|}
\hline \multicolumn{1}{|c|}{ Texto fuente } & \multicolumn{1}{c|}{ Comentario escrito digital } \\
\hline \#INSTATEXTOS: UN ESTUDIO SOBRE LA & $\begin{array}{l}\text { Me parece más pertinente que diga: un } \\
\text { estudio lingüístico de Instagram o un } \\
\text { LINGÜÍSTICA DE INSTAGRAM } \\
\text { estudio de instagram desde la lingüística } \\
\text { textual. } \\
\text { (Ponencia 4, 2016; versión 6, CED 60). }\end{array}$ \\
\hline
\end{tabular}

\section{FOCO}

\section{Coherencia global (Partes canónicas)}

\begin{tabular}{|l|l|}
\hline \multicolumn{1}{|c|}{ Texto fuente } & \multicolumn{1}{|c|}{ Comentario escrito digital } \\
\hline $\begin{array}{l}\text { (...) Pero no existen estudios que relacionen los temas de } \\
\text { publicidad y lectura, considerando a este género como un } \\
\text { factor que influya en la vida de los adolescentes para } \\
\text { promover la lectura, tal es así que nos abocaremos a ocupar } \\
\text { este nicho vacante. }\end{array}$ & $\begin{array}{l}\text { Quedó muy bien la conformación del } \\
\text { nicho de investigación”. }\end{array}$ \\
(Ponencia 1, 2015; versión 2, CED 14)
\end{tabular}

\section{Coherencia global (Articulación entre partes)}

\section{Texto fuente}

Ahora, si bien existe cierta cantidad de artículos sobre el tema y se plantean una variedad de interrogantes, teorías y suposiciones, la cuestión no ha sido abordada hasta el momento desde esta perspectiva (narrativa/literaria) a fondo.

\section{Comentario escrito digital}

A esta información yo la pondría antes que el otro fragmento resaltado; esto vendría a ser el establecimiento del centro y el de arriba ocupación del centro.

(Ponencia 1, 2016; versión 1, CED 6) 
Coherencia local (Registro académico - Uso de terminología específica)

\begin{tabular}{|l|l|}
\hline \multicolumn{1}{|c|}{ Texto fuente } & \multicolumn{1}{c|}{ Comentario escrito digital } \\
\hline Desde una concepción más moderna y científica, para la & Dejen solamente procesos cognitivos; \\
cultura contemporánea leer significa comprender, es decir & destreza está connotado peyorativamente \\
desarrollar varias destrezas mentales o procesos cognitivos & por su adscripción al paradigma \\
(Cassany, 2006) & conductista. \\
& (Ponencia 1, 2015; versión 1, CED 17) \\
\hline
\end{tabular}

\section{Coherencia local (Registro académico - Posicionamiento enunciativo)}

\begin{tabular}{|l|l|}
\hline \multicolumn{1}{|c|}{ Texto fuente } & \multicolumn{1}{c|}{ comentario escrito digital } \\
\hline "Machetes", ¿cuántas veces al escuchar esta & Todavía me parece que tiene un tono muy \\
palabra y uno se remonta a la época secundaria & subjetivo. Les sugiero algo de este estilo: Como \\
con picardía y nostalgia? ¿Quién no los usó nunca? & sabemos los machetes tradicionalmente están \\
& asociados al nivel secundario y se considera una \\
& práctica extendida en su gran mayoría por quienes \\
& transitan o transitaron por este nivel. \\
& (Ponencia 6, 2016; versión 2, CED 2) \\
\hline
\end{tabular}

\section{Resultados}

En relación con los objetivos propuestos, en la Tabla 1, se recogen los tipos de CEDs usados por el docente en las cohortes 2015 y 2016, en porcentajes y números absolutos, para mostrar qué clase de comentarios son más recurrentes en el corpus analizado.

Luego, en las Tablas 2 (cohorte 2015) y 3 (cohorte 2016), se presentan en cada caso los datos cuantitativos de dos grupos (escritores novatos y expertos), a fin de identificar si el tutor formula diferentes tipos de CEDs, en función de estos distintos perfiles, y observar si, más allá de estas diferencias, pueden detectarse similitudes en relación con los desafíos que plantea la complejidad de la escritura académica. 


\subsection{Modo y foco en los comentarios escritos digitales}

Tabla 1. Modo y foco en los comentarios escritos digitales.

\begin{tabular}{|c|c|c|c|c|c|c|}
\hline \multicolumn{5}{|c|}{ PONENCIAS ESTUDIANTILES } & $\begin{array}{l}\text { AÑO } \\
2015\end{array}$ & $\begin{array}{l}\text { AÑO } \\
2016\end{array}$ \\
\hline \multirow{8}{*}{$\begin{array}{l}\mathrm{M} \\
\mathrm{O} \\
\mathrm{D} \\
\mathrm{O}\end{array}$} & \multirow{8}{*}{ Acto de habla } & \multirow{2}{*}{ Directo } & \multicolumn{2}{|c|}{ Orden } & $\begin{array}{c}190 \\
(35,7 \%)\end{array}$ & $\begin{array}{c}115 \\
(28,8 \%)\end{array}$ \\
\hline & & & \multicolumn{2}{|c|}{ Interrogación } & $\begin{array}{c}27 \\
(5 \%)\end{array}$ & $\begin{array}{c}29 \\
(7,2 \%)\end{array}$ \\
\hline & & \multirow{6}{*}{ Indirecto } & Declaración & Afirmativa & $\begin{array}{c}160 \\
(30,1 \%)\end{array}$ & $\begin{array}{c}149 \\
(37,3 \%)\end{array}$ \\
\hline & & & & Negativa & $\begin{array}{c}26 \\
(4,8 \%) \\
\end{array}$ & $\begin{array}{c}12 \\
(3 \%) \\
\end{array}$ \\
\hline & & & Interrogación & & $\begin{array}{c}24 \\
(4,5 \%) \\
\end{array}$ & $\begin{array}{c}6 \\
(1,5 \%) \\
\end{array}$ \\
\hline & & & Condicional & & $\begin{array}{c}25 \\
(4,7 \%) \\
\end{array}$ & $\begin{array}{c}21 \\
(5,2 \%)\end{array}$ \\
\hline & & & Impersonal & & $\begin{array}{c}16 \\
(3 \%)\end{array}$ & $\begin{array}{c}8 \\
(2 \%)\end{array}$ \\
\hline & & & $\begin{array}{c}\text { Ofrecimiento } \\
\text { de alternativa } \\
\text { discursiva }\end{array}$ & & $\begin{array}{c}63 \\
(11,8 \%)\end{array}$ & $\begin{array}{c}59 \\
(14,7 \%)\end{array}$ \\
\hline \multicolumn{5}{|c|}{ TOTAL (COMENTARIOS SEGÚN EL MODO) } & 531 & 399 \\
\hline \multirow{12}{*}{$\begin{array}{l}\mathrm{F} \\
\mathrm{O} \\
\mathrm{C} \\
\mathrm{O}\end{array}$} & Diagramación & & & & $\begin{array}{c}26 \\
(4,8 \%)\end{array}$ & $\begin{array}{c}27 \\
(6,7 \%)\end{array}$ \\
\hline & \multirow{3}{*}{$\begin{array}{l}\text { Coherencia } \\
\text { global }\end{array}$} & Partes canónicas & & & $\begin{array}{c}28 \\
(5,2 \%)\end{array}$ & $\begin{array}{c}29 \\
(7,2 \%)\end{array}$ \\
\hline & & $\begin{array}{l}\text { Articulación entre } \\
\text { partes }\end{array}$ & & & $\begin{array}{c}30 \\
(5,6 \%)\end{array}$ & $\begin{array}{c}34 \\
(8,5 \%)\end{array}$ \\
\hline & & $\begin{array}{l}\text { Comentarios } \\
\text { críticos }\end{array}$ & & & $\begin{array}{c}38 \\
(7,1 \%)\end{array}$ & $\begin{array}{c}48 \\
(12 \%)\end{array}$ \\
\hline & \multirow{3}{*}{$\begin{array}{l}\text { Coherencia } \\
\text { local }\end{array}$} & $\begin{array}{l}\text { Estructura } \\
\text { gramatical }\end{array}$ & & & $\begin{array}{c}81 \\
(15,2 \%)\end{array}$ & $\begin{array}{c}45 \\
(11,2 \%)\end{array}$ \\
\hline & & Puntuación & & & $\begin{array}{c}41 \\
(7,7 \%) \\
\end{array}$ & $\begin{array}{c}36 \\
(9 \%) \\
\end{array}$ \\
\hline & & Reformulación & & & $\begin{array}{c}86 \\
(16,1 \%) \\
\end{array}$ & $\begin{array}{c}53 \\
(13,2 \%) \\
\end{array}$ \\
\hline & \multirow{4}{*}{$\begin{array}{l}\text { Registro } \\
\text { académico }\end{array}$} & Pautas de citación & & & $\begin{array}{c}116 \\
(21,8 \%) \\
\end{array}$ & $\begin{array}{c}73 \\
(18,2 \%) \\
\end{array}$ \\
\hline & & $\begin{array}{l}\text { Uso adecuado de } \\
\text { terminología general }\end{array}$ & & & $\begin{array}{c}29 \\
(5,4 \%) \\
\end{array}$ & $\begin{array}{c}13 \\
(3,2 \%) \\
\end{array}$ \\
\hline & & $\begin{array}{c}\text { Uso adecuado de } \\
\text { terminología } \\
\text { específica }\end{array}$ & & & $\begin{array}{c}8 \\
(1,5 \%)\end{array}$ & $\begin{array}{c}2 \\
(0,5 \%)\end{array}$ \\
\hline & & $\begin{array}{c}\text { Posicionamiento } \\
\text { enunciativo }\end{array}$ & & & $\begin{array}{c}4 \\
(0,7 \%) \\
\end{array}$ & $\begin{array}{c}6 \\
(1,5 \%) \\
\end{array}$ \\
\hline & Ortografía & & & & $\begin{array}{c}44 \\
(8,2 \%)\end{array}$ & $\begin{array}{c}33 \\
(8,2 \%)\end{array}$ \\
\hline \multicolumn{5}{|c|}{ TOTAL (COMENTARIOS SEGÚN EL FOCO) } & 531 & 399 \\
\hline
\end{tabular}

Del análisis de los datos, se desprende que hay tipos de comentarios que son más recurrentes que otros, en ambos corpus. $\mathrm{Si}$ atendemos a la caracterización de los 
comentarios según el 'modo', prevalecen los formulados mediante actos de habla directos de orden. En las ponencias 2015, se registran 190 CEDs de orden; lo que corresponde a un 35,7\% del total de CEDs realizados en las seis ponencias de esta cohorte (531 CEDs). En orden de importancia, le siguen los CEDs indirectos de tipo declarativo afirmativo $(160 ; 30,1 \%)$ y los CEDs de ofrecimiento de una alternativa discursiva $(63 ; 11,8 \%)$.

Esta tendencia se invierte levemente en la cohorte 2016. De los 399 CEDs en las seis ponencias de esta cohorte, 149 corresponden a CEDs de actos de habla indirectos de carácter declarativo afirmativo $(37,3 \%$ del total de los CEDs de estas seis ponencias). Los CEDs de actos de habla directos de orden son 115 (28,8\%) y le siguen en tercer lugar, 59 CEDs de ofrecimiento de alternativa discursiva $(14,7 \%)$.

Si tenemos en cuenta los CEDs según el 'foco', en el año 2015, encontramos mayor número de comentarios a nivel local, referidos a las formas de citado (116 CEDs; $21,8 \%$ ) y a la reformulación a nivel de coherencia local (86 CEDs; 16,1\%). En el año 2016, los porcentajes son similares, ya que la mayor cantidad de CEDs según el foco corresponde a las pautas de citado $(73 \mathrm{CEDs} ; 18,2 \%)$ y a la reformulación a nivel local (53 CEDs; $13,2 \%)$.

\subsection{Comentarios escritos digitales y perfiles de escritor}

Con respecto a los tipos de CEDs elegidos por el profesor para dar retroalimentación a sus estudiantes, hemos notado que habría diferencias, según se trate de perfiles de escritor novatos o expertos. Para ilustrar este aspecto, tomamos de cada año académico dos grupos, uno experto ( $\mathrm{G} 2$ de 2015 y G 2 de 2016) y otro novato (G3 de 2015 y G 5 de 2016).

Ciertas diferencias entre estos dos grupos, en ambas cohortes, están relacionadas con la cantidad y el tipo de CEDs, tal como podemos ver en la siguiente Tabla. 
Tabla 2. Comentarios escritos digitales y perfiles de escritor (grupos expertos y novatos). Ponencias 2015.

\begin{tabular}{|c|c|c|c|c|c|c|}
\hline \multicolumn{5}{|c|}{$\begin{array}{c}\text { PONENCIAS } 2015 \\
\text { GRUPOS EXPERTOS Y NOVATOS }\end{array}$} & $\begin{array}{c}\text { G2 } \\
\text { EXPERTO }\end{array}$ & $\begin{array}{c}\text { G3 } \\
\text { NOVATO }\end{array}$ \\
\hline \multirow{4}{*}{ M } & & \multirow{2}{*}{ Directo } & Orden & & 35 & 37 \\
\hline & & & Interrogación & & 4 & 7 \\
\hline & & \multirow{6}{*}{ Indirecto } & Declaración & Afirmativa & 8 & 76 \\
\hline & & & & Negativa & 2 & 6 \\
\hline $\mathrm{O}$ & & & Interrogación & & 6 & 3 \\
\hline \multirow[t]{2}{*}{$\mathrm{D}$} & & & Condicional & & 2 & 3 \\
\hline & & & Impersonal & & 1 & 4 \\
\hline $\mathrm{O}$ & & & $\begin{array}{c}\text { Ofrecimiento } \\
\text { de alternativa } \\
\text { discursiva }\end{array}$ & & 2 & 10 \\
\hline \multicolumn{5}{|c|}{ TOTAL (COMENTARIOS SEGÚN EL MODO) } & 60 & 146 \\
\hline \multirow{6}{*}{$\mathrm{F}$} & Diagramación & & & & 3 & 5 \\
\hline & \multirow{3}{*}{$\begin{array}{l}\text { Coherencia } \\
\text { global }\end{array}$} & Partes canónicas & & & 0 & 6 \\
\hline & & $\begin{array}{l}\text { Articulación } \\
\text { entre partes }\end{array}$ & & & 9 & 2 \\
\hline & & $\begin{array}{c}\text { Comentarios } \\
\text { críticos }\end{array}$ & & & 1 & 9 \\
\hline & \multirow{3}{*}{$\begin{array}{l}\text { Coherencia } \\
\text { local }\end{array}$} & $\begin{array}{l}\text { Estructura } \\
\text { gramatical }\end{array}$ & & & 7 & 26 \\
\hline & & Puntuación & & & 1 & 26 \\
\hline $\mathrm{O}$ & & Reformulación & & & 9 & 21 \\
\hline \multirow{5}{*}{ C } & \multirow{4}{*}{$\begin{array}{l}\text { Registro } \\
\text { académico }\end{array}$} & $\begin{array}{l}\text { Pautas de } \\
\text { citación }\end{array}$ & & & 22 & 21 \\
\hline & & $\begin{array}{c}\text { Uso adecuado } \\
\text { de terminología } \\
\text { general }\end{array}$ & & & 2 & 7 \\
\hline & & $\begin{array}{c}\text { Uso adecuado } \\
\text { terminología } \\
\text { específica }\end{array}$ & & & 1 & 1 \\
\hline & & $\begin{array}{c}\text { Posicionamiento } \\
\text { enunciativo }\end{array}$ & & & 0 & 2 \\
\hline & Ortografía & & & & 5 & 20 \\
\hline \multicolumn{5}{|c|}{ TOTAL (COMENTARIOS SEGÚN EL FOCO) } & 60 & 146 \\
\hline
\end{tabular}

Como puede observarse con respecto a la cantidad de comentarios, en el año 2015, los CEDs dirigidos al grupo experto son en total 60; en cambio, en el grupo novato la cifra alcanza los 146 CEDs. Es decir, el número de CEDs en las ponencias de los escritores novatos representa más del doble de la cantidad de los realizados en las ponencias de los escritores expertos.

En cuanto al 'modo' de los CEDs, no se observan diferencias en los actos directos de orden (35 y 37), pero sí en los actos indirectos declarativos afirmativos (8, en G2 y 76 , en G3). De manera similar, también se registra cierta diferencia en los comentarios que apuntan a ofrecer una alternativa discursiva (2, en G2 y 10, en G3). 
En relación con los CEDs que hacen hincapié en el 'foco', el número es mayor en todas las dimensiones consideradas en el grupo novato, excepto en la diagramación (3, en $G 2$ y 5 , en $G 3$ ) y en las pautas de citación (22, en $G 2$ y 21, en G3), con cierta paridad. Por otro lado, en los CEDs que se refieren a la articulación entre partes, los números son levemente superiores en el grupo experto (9, en G2 y 2, en G3).

En las categorías referidas a la coherencia global, se observan mínimas diferencias en cuanto a los CEDs sobre las partes canónicas (ningún CED en el grupo experto y 6 en el grupo novato) y sobre los comentarios críticos (1, en G 2 y 9, en G 3). En lo que concierne a la coherencia local, los números son menores en el grupo experto, con respecto al grupo novato, en lo referido a la estructura gramatical (7 y 26); puntuación (1 y 26); reformulación (9 y 21) y ortografía (5 y 20).

En la Tabla 3, presentamos los valores correspondientes al grupo experto y novato de la cohorte 2016. 
Tabla 3. Comentarios digitales y perfiles de escritor (grupos expertos y novatos). Ponencias 2016.

\begin{tabular}{|c|c|c|c|c|c|c|}
\hline \multicolumn{5}{|c|}{$\begin{array}{c}\text { PONENCIAS } 2016 \\
\text { GRUPOS EXPERTOS Y NOVATOS }\end{array}$} & $\begin{array}{c}\text { G2 } \\
\text { EXPERTO }\end{array}$ & $\begin{array}{c}\text { G5 } \\
\text { NOVATO }\end{array}$ \\
\hline \multirow[b]{3}{*}{ M } & & \multirow{2}{*}{ Directo } & Orden & & 14 & 31 \\
\hline & & & Interrogación & & 11 & 7 \\
\hline & & \multirow{6}{*}{ Indirecto } & Declaración & Afirmativa & 25 & 36 \\
\hline \multirow{2}{*}{$\mathrm{O}$} & & & & Negativa & 0 & 7 \\
\hline & & & Interrogación & & 2 & 1 \\
\hline \multirow{2}{*}{$\mathrm{D}$} & & & Condicional & & 4 & 3 \\
\hline & & & Impersonal & & 1 & 0 \\
\hline $\mathrm{O}$ & & & $\begin{array}{c}\text { Ofrecimiento } \\
\text { de alternativa } \\
\text { discursiva }\end{array}$ & & 2 & 19 \\
\hline \multicolumn{5}{|c|}{ TOTAL (COMENTARIOS SEGÚN EL MODO) } & 59 & 104 \\
\hline \multirow{6}{*}{$\mathrm{F}$} & Diagramación & & & & 4 & 8 \\
\hline & \multirow{3}{*}{$\begin{array}{l}\text { Coherencia } \\
\text { global }\end{array}$} & Partes canónicas & & & 1 & 5 \\
\hline & & $\begin{array}{l}\text { Articulación } \\
\text { entre partes }\end{array}$ & & & 3 & 6 \\
\hline & & $\begin{array}{l}\text { Comentarios } \\
\text { críticos }\end{array}$ & & & 5 & 24 \\
\hline & \multirow{3}{*}{$\begin{array}{l}\text { Coherencia } \\
\text { local }\end{array}$} & $\begin{array}{l}\text { Estructura } \\
\text { gramatical }\end{array}$ & & & 14 & 11 \\
\hline & & Puntuación & & & 3 & 8 \\
\hline $\mathrm{O}$ & & Reformulación & & & 7 & 12 \\
\hline \multirow{5}{*}{$\begin{array}{l}\mathrm{C} \\
\mathrm{O}\end{array}$} & \multirow{4}{*}{$\begin{array}{l}\text { Registro } \\
\text { académico }\end{array}$} & $\begin{array}{l}\text { Pautas de } \\
\text { citación }\end{array}$ & & & 15 & 22 \\
\hline & & $\begin{array}{l}\text { Uso adecuado de } \\
\text { terminología } \\
\text { general }\end{array}$ & & & 3 & 1 \\
\hline & & $\begin{array}{l}\text { Uso adecuado } \\
\text { terminología } \\
\text { específica }\end{array}$ & & & 0 & 0 \\
\hline & & $\begin{array}{c}\text { Posicionamiento } \\
\text { enunciativo }\end{array}$ & & & 0 & 0 \\
\hline & Ortografía & & & & 4 & 7 \\
\hline \multicolumn{5}{|c|}{ TOTAL (COMENTARIOS SEGÚN EL FOCO) } & 59 & 104 \\
\hline
\end{tabular}

$\mathrm{Al}$ igual que en el año 2015, se observa menor número total de comentarios en la ponencia del grupo experto (59 CEDs), que en la del grupo novato (104 CEDs).

En relación con los CEDs de 'modo', hay menor cantidad de CEDs de orden en el grupo experto que en el novato (14, en G2 y 31, en G5), de actos indirectos declarativos afirmativos (25, en $G 2$ y 36, G 5) y de ofrecimiento de alternativa discursiva (2, en $\mathrm{G} 2$ y 19 , en $\mathrm{G} 5$ ).

En cuanto a los CEDs de 'foco', se repiten las diferencias en relación con menor cantidad de CEDs en el grupo experto (G 2), con respecto al novato (G 5), tanto a nivel de coherencia global como local: diagramación (4, en $G 2$ y 8, en G 5); partes canónicas (1 y 5, respectivamente); comentarios críticos (5 y 24); puntuación (3 y 8); reformulación (7 y 12); pautas de citación (15 y 22) y ortografía (4 y 7). En cambio, no 
se observan diferencias en los CEDs referidos al uso adecuado de terminología general (3, en G 2 y 1, en G 5) y a la estructura gramatical (14, en G2 y 11, en G 5).

Con respecto a la articulación entre partes, a diferencia de la cohorte 2015 se registra un poco de CEDs en el grupo novato que en el experto (6 y 3), y para el uso de las pautas de citación, se observa una leve diferencia entre el grupo experto y novato (15 CEDs, en G 2 y 22 CEDs, G 5); es decir, habría una pequeña variación con respecto a los casos de 2015, en los que se observa una mayor paridad en el número de CEDs referidos a este aspecto.

\section{Discusión}

Del análisis global de los resultados obtenidos con respecto a los tipos de CEDs docentes, podemos decir que se observan resultados similares en ambas cohortes, atendiendo las categorías de 'modo' y 'foco'.

Desde el punto de vista del 'modo' de los CEDs, los más empleados son los actos directos de orden y, con respecto a los actos indirectos, los declarativos afirmativos y los que ofrecen una alternativa discursiva. Aunque los CEDs de orden aparecen en primer término en la cohorte 2015 y, en segundo lugar, en la cohorte 2016, resulta relevante que sea el más usado por el docente. El predomino de este tipo de comentarios está en consonancia con los hallazgos de Cuevas, Mellado, Rodríguez, Valenzuela y Vásquez (2017), quienes arribaron a estos mismos resultados al observar los tipos de CEDs implementados por docentes en dos establecimientos secundarios chilenos. Señalan que esta actitud directiva es valorada positivamente por los estudiantes, pues aprovechan mejor los comentarios cuando se les dice puntualmente qué aspecto del texto deben corregir.

Si relacionamos estos datos con los postulados del modelo de revisión de Scardamalia y Bereiter (1983), en cuanto a las categorías 'comparar, diagnosticar y operar', podemos inferir la importancia del tipo de retroalimentación que proporciona el docente del corpus analizado, en términos de mayor 'andamiaje' (Bruner, 1984) en dos sentidos: proporcionar mayor cantidad de CEDs a los grupos novatos y mayor cantidad de CEDs directivos, con respecto a otras posibilidades. Esto pone de manifiesto que el docente, ante la inexperiencia de sus estudiantes en escritura académica, ayuda a comparar el texto producido con los propios objetivos de producción, visibilizando la tensión entre estos y las restricciones genéricas y contextuales, teniendo en cuenta lo complejo que es para los estudiantes principiantes iniciarse en las prácticas académicas propias del contexto universitario. De este modo, particularmente, dirige la atención de los novatos para que identifiquen los aspectos a modificar y operen los cambios necesarios (Scardamalia \& Bereiter, 1983), bajo dos modalidades: detección de determinado problema y prescripción específica del cambio (actos directos de orden), o identificación y sugerencia de posibilidades para resolver estos problemas (ofrecimiento de alternativa discursiva). 
En cuanto a la categoría de 'foco', si bien los CEDs se dirigen hacia los diferentes aspectos formales y de coherencia global y local, hemos encontrado un porcentaje levemente mayor en los CEDs referidos a las pautas de citación: en 2015, 116 CEDs (21,8\%), y en 2016, 73 CEDs (18,2\%). Estos resultados están en consonancia con lo que constituye uno de los propósitos que persiguen los CEDs: dar orientaciones para que los estudiantes principiantes, en tanto escritores inexpertos, puedan ingresar a su comunidad de práctica mediante la realización de ajustes necesarios para que el texto producido resulte aceptable dentro de esta. En concordancia con esto, Natale (2013) da cuenta de una investigación sobre diversas estrategias discursivas implementadas por docentes universitarios de diferentes disciplinas, a fin de que los estudiantes puedan acceder a las convenciones del género, los desarrollos teóricos y la metodología del área de referencia. Entre las ocho estrategias determinadas, en función de su corpus, llega a la conclusión de que una de las más usadas por los profesores, es la estrategia denominada 'guiar para la realización de un texto' que constituye un andamiaje importante para la producción textual. Esta ayuda se materializa mediante 'órdenes' que indican que se deben introducir cambios en el texto, ya se trate de reformulaciones o eliminación de algún segmento textual. Estas 'órdenes', lejos de ser consideradas como tales, pueden ser entendidas como textos instructivos que dirigen el proceso de escritura; es decir, el docente señala con precisión qué modificaciones es necesario incluir, a fin de que el texto pueda ser evaluado como un ejemplar aceptable en la comunidad discursiva en la que se inserta.

\section{CONCLUSIONES}

En el contexto de las investigaciones sobre escritura académica en los estudios superiores, este trabajo busca aportar algunos resultados para la discusión sobre alternativas posibles para ocuparse de ella en las aulas universitarias. En esta oportunidad, hemos focalizado en los comentarios escritos digitales (CEDs), entendidos como uno de los recursos posibles de las prácticas de retroalimentación, provistos por un docente a las distintas versiones de las ponencias grupales de estudiantes principiantes de Humanidades de una universidad pública argentina, en el marco de ciclos de investigación-acción. Hemos seleccionado doce ponencias (con un promedio de seis versiones cada una) de dos cohortes (2015 y 2016), a fin de testear como hipótesis la posible relación entre la cantidad y el tipo de CEDs proporcionados por el docente y diferentes perfiles de escritor, en relación con el grado de dominio de la escritura a nivel general (coherencia global y local, y aspectos normativos) y a nivel de las restricciones específicas del discurso académico.

En función de los objetivos planteados, hemos llegado a las siguientes conclusiones:

- En cuanto a las clases de CEDs más frecuentes en el corpus analizado, hemos observado que el profesor recurre en mayor medida a los CEDs directivos, lo 
que resulta bastante razonable tratándose de estudiantes principiantes que recién ingresan a una comunidad disciplinar, con modos distintivos de producir y comunicar conocimiento científico. Cabe destacar que se trata de CEDs específicos que brindan directivas puntuales, en relación con las distintas dimensiones del discurso académico y, por tanto, son CEDs adaptados a las necesidades de estos estudiantes noveles que requieren de apoyos sistemáticos y oportunos, hasta poder alcanzar la autorregulación de los procesos de escritura académica.

- En relación con esto, esta intención del docente de proporcionar comentarios contingentes a las necesidades de sus estudiantes se pone de manifiesto, en mayor medida, en el caso de los perfiles de escritor novato, con respecto a los expertos, ya que aquellos precisan orientaciones no solo con respecto a las restricciones propias del discurso académico, sino también directivas vinculadas con el dominio de la escritura a nivel general, atendiendo a sus aspectos globales, locales y normativos.

- Sin embargo, más allá de estas diferencias, se han apreciado similitudes en los CEDs, en relación con los diferentes desafíos que plantea la complejidad de la escritura académica. En este sentido, en las ponencias de ambos perfiles de escritor se han observado cantidades semejantes de CEDs referidos a un aspecto central del discurso académico, como es el de las pautas formales de citación.

Con respecto a las limitaciones de este estudio, destacamos que, si bien el corpus analizado ha permitido dar cuenta de diferentes aristas involucradas en las prácticas de retroalimentación, aún no ha sido explotado en todas sus potencialidades. Resta analizar en qué medida los estudiantes aprovechan los CEDs proporcionados por el docente en los diversos borradores, para una franca mejora en la calidad de sus versiones finales, y ponderar qué clases de CEDs contribuyen a la mejora global de los escritos, teniendo en cuenta a la vez los diferentes perfiles de escritor. Asimismo, cabe señalar que las conclusiones a las que hemos arribado, a partir de los resultados obtenidos solo son válidas para el corpus analizado. En tal sentido, deberían ser contrastadas en mayor cantidad de ejemplares textuales revisados por el mismo docente en otras cohortes, y en escritos revisados por otros docentes.

Por otro lado, en el marco de los nuevos paradigmas dialógicos de retroalimentación, el gran desafío es avanzar en las investigaciones sobre revisión colaborativa entre pares, mediante herramientas metodológicas que permitan indagar en profundidad las potencialidades cognitivas de esta construcción conjunta de dar y recibir comentarios (Nicol, 2010), bajo el supuesto de que los estudiantes asumirán una mayor responsabilidad y un rol más activo en los procesos autorregulatorios de aprendizaje. 


\section{REFERENCIAS BIBLIOGRÁFICAS}

Aguilera, M. \& Boatto, Y. (2013). Seguir escribiendo... seguir aprendiendo: La escritura de textos académicos en el nivel universitario. Zona Próxima, 18, 136145.

Álvarez Angulo, T. (2011). Revisar y reescribir textos académicos en la formación del profesorado. Revista Complutense de Educación, 22(2), 269-294.

Alvira, R. (2016). The impact of oral and written feedback on efl writers with the use of screencasts. Profile. Issues in Teachers' Professional Development, 18(2), 79-92.

Bajtín, M. (1982). Estética de la creación verbal. México: Siglo XXI editores.

Bazerman, C. (2004). Speech acts, genres, and activity systems: How texts organize activity and people. En P. Prior \& C. Bazerman (Eds.), What writing does and how it does it (pp. 309-339). Nueva Jersey: Lawrence Erlbaum.

Bazerman, Ch., Little, J., Bethel, L., Chavkin, T., Fouquette, D. \& Garufis, J. (2005). Reference guide to writing across the curriculum. West Lafayette, Indiana: Parlor Press.

Bruffee, K. (1998). Collaborative learning: Higher education, interdependence, and the authority of knowledge. Londres: The John Hopkins University Press.

Boucher, C. (2015). La rétroaction: Un élément phare pour l'apprentissage de nos étudiants. Le Tableau, 4(3) [en línea]. Disponible en: http://pedagogie.uquebec.ca/portail/system/files/documents/membres/leta bleau-v4-n3 2015.pdf

Bruner, J. (1984). Acción, pensamiento y lenguaje. Barcelona: Paidós.

Cano, M., Corcelles, M., Castelló, M. \& Fuentealba, M. (2012). Características y funciones de las ayudas en la revisión colaborativa de textos científicosacadémicos, Congrés Iinternaciona de Docéncia Universitária Innovació, 1(1).

Carlino, P. (2010). Reading and writing in the Social Sciences in Argentine universities. En Ch. Bazerman, R. Krut, K. Lunsford, S. McLeod, S. Null, P. Rogers \& A. Stansell (Eds.), Traditions of Writing Research (pp. 283-296). Nueva York: Routledge/Taylor and Francis.

Carlino, P. (2013). Alfabetización académica diez años después. Revista Mexicana de Investigación Educativa, 18(57), 355-381.

Case, S. (2007). Reconfiguring and realigning the assessment feedback processes for an undergraduate criminology degree. Assessment \& Evaluation in Higher Education, 32(3), 285-299. 
Corcelles, M. \& Oliva, A. (2016). La escritura colaborativa de textos académicos: Un proyecto en el aula de Psicología de la Educación. En G. Bañales, M. Castelló \& N. Vega López (Eds.), Enseñar a leer y a escribir en la educación superior. Propuestas educativas basada en la investigación (pp. 129-158). México DF: SM México y Univ. Autónoma de Tamaulipas.

Corcelles, M., Cano, M., Bañales, G. \& Vega, N. (2013). Enseñar a escribir textos científico-académicos mediante la revisión colaborativa: El trabajo final de grado en Psicología. Revista de docencia universitaria, 11(1), 79-104.

Corcelles, M., Cano, M., Mayoral, M. \& Castelló, M. (2017). Enseñar a escribir un artículo de investigación mediante la revisión colaborativa: Percepciones de los estudiantes. Revista Signos. Estudios de Lingüistica, 50(95), 337-360.

Creme, P. \& Lea, M. (2000). Escribir en la universidad, Barcelona: Gedisa.

Cuevas, D., Mellado, A., Rodríguez, J., Valenzuela, E. \& Vásquez, J. (2017). Comentarios escritos a ensayos producidos por estudiantes de tercer y cuarto año medio de dos liceos de la provincia de Concepción y respuestas de los estudiantes al feedback del docente. Tesis de grado Universidad Católica de la Santísima Concepción, Concepción, Chile.

Duijnhouwer, H. (2010). Feedback effects on student's writing motivation, process and performance. Países Bajos: Dutch Interuniversity Centre for Educational Research.

Ferris, D. (1999). The case for grammar correction in 12 writing classes. A response to Truscott (1996). Journal of Second Language Writing, 8(1), 1-11.

Flower, L. (1994). The construction of negotiated meaning: A social cognitive theory of writing. Carbondale, IL: Southern Illinois University Press.

Flower, L. \& Hayes, J. (1981). A cognitive process theory of writing. College Composition and Communication, 32, 365- 87.

García-Yeste, M. (2013). Electronic feedback: Pedagogical considerations for the implementation of software. The EuroCALL Review, 21(2), 1-6.

Hayes, J. (1995). Un nouveau modèle du processus d'écriture. En J.-Y. Boyer, J.-P. Dionne \& P. Raymond (Dirs.), La production de textes, vers un enseignement de l'écriture (pp. 49-72). Montréal: Logiques.

Kemmis, S. (2009). Action research as a practice-changing practice. Educational Action Research, 17(3), 463-474. 
Lillo Durán, J. \& Ferreira Cabrera, A. (2014). Un modelo teórico-metodológico para investigación empírica en feedback correctivo escrito en una segunda lengua. Onomázein, 30, 90-110.

Maxwell, J. (2012). A realist approach for Qualitative Research. Thousand Oaks, CA: Sage.

Molina Natera, V. (2015). Panorama de centros y programas de escritura en Latinoamérica. Cali, Colombia: Sello Editorial Javeriano.

Natale, L. (2013). Relaciones intersubjetivas entre expertos y novatos en devoluciones escritas de docentes universitarios. Legenda, 17(16), 86-114.

Nicol, D. (2010). From monologue to dialogue: Improving written feedback processes in mass higher education. Assessment \& Evaluation in Higher Education, 35(5), 501-517.

Nicol, D. \& Macfarlane-Dick, D. (2006). Formative assessment and selfregulated learning: A model and seven principles of good feedback practice. Studies in Higher Education, 31(2), 199-218.

Nicol, D., Thomson, A. \& Breslin, C. (2014). Rethinking feedback practices in higher education: A peer review perspective. Assessment \& Evaluation in Higher Education, 39(1), 102-122.

Orsmond, P. \& Merry, S. (2009). Processing tutor feedback: A consideration of qualitative differences in learning outcomes for high and non-high achieving students. Ponencia presentada en el Fostering Communities of Learners, 13th EARLI conference, August 25-29, Ámsterdam, Alemania.

Pardo, M. \& Castelló, M. (2016). Teaching writing for learning at university: A proposal based on collaborative review. Infancia y Aprendizaje. Journal for the Study of Education and Development, 39, 560-591.

Parodi, G. (Ed.) (2010). Alfabetización académica y profesional en el Siglo XXI: Leer y escribir desde las disciplinas. Santiago de Chile: Ariel.

Pérez Abril, M. \& Rincón Bonilla, G. (2013). Para qué se lee y escribe en la universidad colombiana. Un aporte a la consolidación de la cultura académica del país. Bogotá: Pontificia Universidad Javeriana.

Peterson, S. (2010). Améliorer l'expression écrite des élèves grâce à la rétroaction des enseignants, Faire la différence: De la recherche à la pratique, 1-4 [en línea]. Disponible en: http://www.edu.gov.on.ca/fre/literacynumeracy/inspire/research/WW_Stud ent_Writing_French.pdf 
Russell, D. (2012). Écrits universitaires / écrits professionnalisants / écrits professionnels: Est-ce qu'«écrire pour apprendre » est plus qu'un slogan ?. Pratiques, 153-154, 21-34.

Scardamalia, M. \& Bereiter, C. (1983). The development of evaluation, diagnostic and remedial capabilities in children's composing. En M. Martlew (Dir.), The psychology of written language, Development and educational perspectives (pp. 67-96). Chichester: John Wiley and Sons.

Scardamalia, M. \& Bereiter, C. (1992). Dos modelos explicativos de los procesos de producción escrita. Infancia y aprendizaje, 58, 43-64.

Straub, R. \& Lunsford, R. (1995). Readers reading. Responding College Student Writing, Cresskill: Hampton.

Tapia-Ladino, M. (2014). Los comentarios escritos: Género orientado a la consecución de otro género en el proceso de la escritura académica. Onomázein, 30, 254-268.

Tapia-Ladino, M., Bustos, C., Salazar, T., Muñoz, C., Varela, M. \& Sáez, K. (2014). Reacciones de los estudiantes a los comentarios escritos que proveen docentes de lenguaje en enseñanza secundaria chilena. Enunciación, 19(1), 3952.

Tapia-Ladino, M., Arancibia, B. \& Correa, R. (2016). Rol de los comentarios escritos en la construcción de la tesis desde la perspectiva de estudiantes tesistas y profesores guía. Universitas Psychologica, 15(4), 1-14.

Truscott, J. (1996). The case against grammar correction in 12 writing classes. Language Learning, 46(2), 327-369.

Tuzi, F. (2004). The impact of e-feedback on the revisions of L2 writers in an academic writing course. Computers and Composition, 21(2), 217-235.

Zimmerman, B. J. (2000). Attaining self-regulation: A social cognitive perspective. En M. Boekaerts, P. R., Pintrich \& M. Zeidner (Eds.), Handbook of selfregulation (pp. 13-39). San Diego, CA: Academic Press.

\section{NOTAS}

${ }^{1}$ Los resultados de investigación de los movimientos 'Escribir a través del currículum' (WAC) y 'Escribir en las disciplinas' (WID) han permitido configurar propuestas didácticas en las que se escribe para aprender una materia y se aprende a escribir según los géneros propios de un campo del saber (Bazerman, Little, Bethel, Chavkin, Fouquette \& Garufis, 2005). 
2 Esta asignatura pertenece a la carrera de Letras y como contenidos disciplinares, se ofrece a los estudiantes una introducción a los estudios del discurso. Sus ejes conceptuales son: los procesos de comprensión y producción textual; las tipologías discursivas -poniendo énfasis en los estudios sobre argumentación- y el discurso científico-académico.

${ }^{3}$ Por razones de espacio, no ofrecemos ejemplos de todas las categorías.

\section{AGRADECIMIENTOS}

*Agradecemos al CONICET (Consejo Nacional de Investigaciones Científicas y Tecnológicas) y a la SCAIT (Secretaría de Ciencia, Arte e Innovación Tecnológica) de la Universidad Nacional de Tucumán (Argentina). 